\title{
Evolution of Opinions in the Growth-vs-Environment Debate: Extended replicator dynamics
}

\author{
Jeroen C.J.M. van den Bergh ${ }^{1,2,3,4}$, Ivan Savin ${ }^{1,5}$ and Stefan Drews ${ }^{1}$
}

\begin{abstract}
The evolution of opinions in the long-standing debate on growth-versus-environment may affect support for important sustainability policies, in areas such as biodiversity loss, climate change, deforestation and freshwater scarcity. In order to understand this evolution, we develop a model describing the dynamics of four distinct opinions as identified in recent surveys, namely growth-atall-cost, green growth, agrowth and degrowth. The model is based on modifying standard replicator dynamics to match a multi-group structure. Individuals are influenced by local or global interactions with others, based on adjacent opinion groups and exposure to information about environmental change. Psychological resistance to opinion change is also accounted for. The model is calibrated with recent survey data. Numerical analysis shows which opinions survive under particular conditions. We find, among others, that under local interactions, ultimate outcomes are characterized by lack of consensus, i.e. survival of multiple opinions. In addition, equal impacts of environmental change on opinions do not always translate in joint survival of associated opinions. Under worsening environmental conditions while continuing economic growth, opinions shift from green growth to agrowth and degrowth. Fostering global interactions among individuals, causing them to be influenced by a broad spectrum of opinions, makes consensus more likely. We also consider model dynamics if feedback from opinions to policy to environmental change and back is included. This confirms robustness of the results. It should be noted that the model is not meant to predict but to explore the consequences of combinations of assumptions about social networks, psychological mechanisms, environmental dynamics, and connections between opinion distribution and environmental policy. The study represents the first analysis of opinion dynamics in the growth-vsenvironment debate and suggests a number of routes for further investigation.
\end{abstract}

Keywords: opinion dynamics; economic growth; environment; sustainability; climate change.

Highlights: We study opinion dynamics in the growth-versus-environment debate | This dynamics is relevant as it affects support for sustainability policies | Modified replicator dynamics with multiple groups is calibrated on recent survey data / We consider adjacent group and global interactions | The model includes feedback of information about environmental change and policy.

\footnotetext{
${ }^{1}$ Institute of Environmental Science and Technology, Universitat Autònoma de Barcelona, Spain.

${ }^{2}$ ICREA, Barcelona, Spain.

${ }^{3}$ School of Business and Economics \& Institute for Environmental Studies, VU University Amsterdam, the Netherlands.

${ }^{4}$ Corresponding author: ICTA-UAB, Edifici Z, UAB Campus, 08193 Bellaterra, Spain; jeroen.bergh@uab.es, phone: +34-93-5868773.

${ }^{5}$ Graduate School of Economics and Management, Ural Federal University, Yekaterinburg, Russian Federation.
} 


\section{Introduction}

Since the 1950s a debate has been wavering on the natural and social "limits-to-growth", comprising optimists, pessimists and more subtle viewpoints (Galbraith, 1958; Mishan, 1967; Meadows et al., 1972; Maddox, 1972; Beckerman, 1976; Hirsch, 1976; Daly, 1977; Simon, 1981; Arrow et al., 1995; Kahneman et al., 2004; Victor, 2008; Jackson, 2009). Recently, the debate has been revived in academia by the challenge of avoiding dangerous climate change (van den Bergh, 2017), while it also has received frequent attention in major global news outlets (Turner and Alexander, 2014; Porter, 2015). Although the literature on this debate is extensive, surprisingly no attention has been given to associated opinion dynamics. Obtaining insight into such dynamics is relevant as support for particular sustainability policies - such as in the areas of biodiversity loss, climate change, deforestation or freshwater scarcity - may depend on the degree of consensus in the debate. Opinion dynamics are driven by two main factors, namely interactions between individuals with the same, similar and distinct opinions, and information about economic growth and environmental problems through various channels, such as newspapers, television and Internet-based social media. Modelling can help to understand and explore the resulting opinion dynamics under distinct assumptions and scenarios as it involves complex interactions between many individuals participating in overlapping social networks of opinion exchange. Accordingly, one can examine if and when opinion dynamics in a population results in a stable distribution reflecting consensus, fragmentation or polarization.

Political opinions as in the growth debate are associated with worldviews (Drews and van den Bergh, 2017), formed mainly during childhood and adolescence - under the influence of parents, other family, teachers and peers. Relevant insights are provided by social psychological research (Johnston and Wronski, 2013) on the individual perspective behind formation of opinions. It finds that opinion dynamics may be driven by compliance with the majority opinion, peer pressure toward conformity, or a process of deliberation by individuals exchanging facts or opinions (Lorenz et al., 2011). Modelling allows to capture the collective dynamics resulting from the interaction of many individuals (Castellano et al., 2009; Xie and Song, 2016; Sîrbu et al., 2017; Tur et al., 2018). The application of general models is motivated by opinion dynamics in distinct areas sharing basic features, which is due to their basis being the same human capacity for opinion formation and social interaction. Evolutionary modelling emerges as a suitable modelling approach as it typically describes the interaction of many individuals in a population characterised by diversity of behaviours or opinions (Gargiulo and Ramasco, 2012).

Here we develop a simple model of opinion dynamics that reflects the essence of the growthvs-environment debate. It involves an extension of the well-known model of replicator dynamics (Taylor and Jonker, 1978) resulting from describing adjacent interactions among multiple groups. The resulting approach has the advantage of being relatively simple, implying transparency and a limited number of parameters. It is not meant to be predictive but aimed at exploring likely outcomes under distinct assumptions and scenarios. It is clear that the growth debate is too complex and involves too many people to be predictive. However, we can learn about potential changes under particular conditions and assumptions, and this would already be a great advance over the static, descriptive and historical accounts. It allows an analysis of the case in which individuals are influenced by others with sufficiently similar opinions, causing local interactions in the population to steer opinion dynamics. For comparison, we also consider the case of global connections, i.e. interactions between all individuals in the population. In addition, individuals are influenced by (information about) the development of environmental problems over time. We further consider an 
extended model with feedback from opinions to policy to environmental change and back. A few studies provide insight into this (Tjernström and Tietenberg, 2008; Anderson et al., 2017).

Of course, one could follow a more disaggregate modelling method, and describe mutual interactions among individual agents with psychological features and worldviews (a more behavioural approach), which would lead to an agent-based model (ABM). We think it is not a logical place to begin, though, for three reasons: opinion dynamics modelling is dominated by aggregate models (see surveys mentioned above); an ABM approach would require more assumptions regarding parameter values, while not necessarily capturing better the essential factors and dynamics. Given the limited empirical data available in the literature, notably absence of long term time series on opinions in the growth-vs-environment debate, it seems wise to adopt an initial model approach that is simple and transparent with relatively few parameters. This may start a new line of research, which can guide further work and suggest hypotheses for testing by empirical research or confirmation and elaboration through more disaggregate models.

We should stress that our study is not completely aggregate because it includes a subtle disaggregation by innovatively adapting the standard replicator dynamics to four groups with an associated asymmetric network structure. Such an approach comes in fact down to an intermediate level of (dis)aggregation, which allows the merger of certain advantages of aggregate and disaggregate approaches. In addition, one can interpret certain parameters in the model from a behavioural-psychological angle. While opinion dynamics in the growth debate might show similarities to that regarding environmental values or political opinions, the uniqueness of the current study consists of three elements. First, the asymmetric network structure which captures how opinions in the growth-vs-environment debate are positioned from one extreme to another. Second, the heterogeneous effect of environmental dynamics on distinct positions. And third, the initial values of these positions are unique and critical for the final outcome.

\section{Opinion dynamics in the growth debate}

The theme of opinion dynamics has received limited attention in the context of the growth-vsenvironment debate. Schmelzer (2015) traces the historical roots of the 'economic growth paradigm', identifying a series of critical factors, such as GDP accounting, international organisations, World War II, the Cold War and notions of social progress. Earlier, Sandbach (1978) argued that Malthusian and environmentalist perspectives rose to prominence in the 1960s and early 1970s but then witnessed a decline in interest. This he attributes to criticisms of these perspectives and economic and political changes which reduced interest in the environment, including the realization that reducing growth would not just be difficult but potentially very costly. The author describes the events, publications and opinion leaders influencing the course of the debate and associated shifts in opinions. A more recent history is sketched by Higgs (2014), who argues that the strong commitment of modern societies to economic growth has marginalised the ample evidence for the problematic relationship between growth and environmental sustainability.

Research has identified several channels through which environmental concern, notably about climate change, is influenced by experiences of environmental change. Studies show that temperature anomalies - i.e. temperature deviations from the mean - have an effect on various public perceptions of climate change (Capstick et al., 2015). In addition, direct and indirect experiences of extreme weather events can affect public perceptions (Demski et al., 2017). Indirect experiences 
involve TV meteorologists educating their viewers on the links between local extreme weather and climate change (Zhao et al., 2014).

By considering the influence of information on opinions in this study, we effectively examine how psychological features play a role, such as openness versus resistance to other worldviews or new information. One may argue that motivated reasoning leads to politically polarized views on climate change, that is, ideologies and values shape people's interpretation of climate information and subsequent concern (Kahan et al., 2012). More information would then only increase concern if it is consistent with one's worldviews. However, this position is debatable for various reasons. First, the study by Kahan et al. focuses on general knowledge or education, not specifically climate-related information. Second, empirical studies for six culturally and politically diverse countries show that higher levels of knowledge about the consequences (and causes) of climate change are linked to greater concern for the large majority of countries, even when controlling for different values (Shi et al., 2016). We assume that with rising environmental deterioration, the influence of environmental knowledge will take precedence over worldviews. Third, recent research on environmental communication has discovered various ways of bridging polarized environmental views by information provision that is in line with people's values and ideology (Johnson, 2017; Whitmarsh and Corner, 2017). In addition, whereas in a bi-polar case of pro/anti-growth political ideology and worldviews may dominate, in our study with a focus on four opinions this connection is likely to be weaker.

Two recent opinion survey studies about the growth debate among citizens and scientists provide a useful background for the empirical set-up employed in the analysis of this study (Drews and van den Bergh, 2016, 2017). These studies identified four main opinions that go beyond a simple dichotomy of optimism/pessimism or pro/anti-growth: (1) Growth-at-all-cost (GC) with full priority for economic growth and little to no concern for the environment; (2) Green growth (GG) expressing the belief that economic growth and environmental sustainability are desirable and compatible; (3) Agrowth (AG) reflecting the idea that since GDP is not a good proxy of social welfare we should be indifferent about economic (=GDP (per capita)) growth; and (4) Degrowth (DG) reflecting the idea that we need to stop economic growth and downscale the economy to achieve environmental sustainability. Our model of opinion dynamics will describe the shares of these four opinions in the population of all opinions. Table 1 explains these opinions and summarizes the core features of the associated individuals. What is especially attractive of this more subtle distinction as opposed to a classic pro/anti-growth or pessimistic/optimistic-about-growth-limits distinctions is that while a change in these dichotomous opinion settings is difficult - though not impossible - because of ideological barriers, such barriers are weaker between adjacent opinions in the quadruple case. We also considered two variables framed as "I have strong opinion about economic growth" and "I have mixed opinions about economic growth" from an earlier empirical study about the growth debate (Drews and van den Bergh, 2016) as an indicator of attitude strength (Howe and Krosnick, 2017) or resistance to changing one's opinion in the face of new information or other opinions. The two indicators show average scores for GC, GG, AG and DG opinions, on a scale from 1 (totally disagree) to 7 (totally agree), of 4.13, 4.26, 4.24 and 4.33, and respectively $4.184 .20,4.49,4.45$. In addition, no significant difference in the distribution of responses is found across the four opinions. All this indicates that there is no evidence for assuming heterogeneity in this respect, motivating the assumption of equal attitude strength between opinion groups in the model. 
Table 1. Sensitivities of individuals' opinions to economic, environmental and social contexts

\begin{tabular}{|l|l|l|l|l|}
\hline \multirow{2}{*}{ Type of influence } & \multicolumn{3}{|c|}{ Four opinions in the growth-versus-environment debate } \\
\cline { 2 - 5 } & $\begin{array}{l}\text { Growth-at-all-cost } \\
(G C)\end{array}$ & $\begin{array}{l}\text { Green growth } \\
(G G)\end{array}$ & $\begin{array}{l}\text { Agrowth } \\
(A G)\end{array}$ & $\begin{array}{l}\text { Degrowth } \\
(D G)\end{array}$ \\
\hline Economic growth & $\begin{array}{l}\text { Strong positive } \\
\text { opinion about GDP } \\
\text { (per capita) growth }\end{array}$ & $\begin{array}{l}\text { Weak positive } \\
\text { opinion about GDP } \\
\text { (per capita) growth }\end{array}$ & $\begin{array}{l}\text { GDP (per capita) } \\
\text { ignored }\end{array}$ & $\begin{array}{l}\text { Negative opinion } \\
\text { about GDP (per } \\
\text { capita) growth }\end{array}$ \\
\hline Environment & Largely ignored & Moderate focus & $\begin{array}{l}\text { Moderate-strong } \\
\text { focus }\end{array}$ & Strong focus \\
\hline Social network & $\begin{array}{l}\text { Strong with GC } \\
\text { Weak with GG }\end{array}$ & $\begin{array}{l}\text { Strong with GG } \\
\text { Weak with GC, AG }\end{array}$ & $\begin{array}{l}\text { Strong with AG } \\
\text { Weak with GG, DG }\end{array}$ & $\begin{array}{l}\text { Strong with DG } \\
\text { Weak with AG }\end{array}$ \\
\hline
\end{tabular}

Table 1 shows that with respect to sensitivities to real-world changes, individuals in the first group with opinion GC are unaffected by environmental changes. Although one might think this view is extreme, Drews and van den Bergh (2016) find that a small proportion of individuals actually fall into this category. Other research suggests that associated environmental disinterest and skepticism are due to insufficient education and environmental knowledge, religious and conservative values, lack of trust in science and society, and other concerns (e.g., economic success and growth) that are seen as competing with environmental concerns (Zhou, 2015). On the other end of the spectrum, we assume that DG shows the highest sensitivity to environmental change. This is in line with findings from Drews and van den Bergh (2016) that support of degrowth is significantly associated with environmental values. The two remaining groups GG and AG fall in-between. A network structure of diffusion of opinions about growth-versus-environment reflects a strong degree of homophily, meaning that individuals tend to interact mainly with those holding similar opinions (Shalizi and Thomas, 2011). The next section will offer more details about the social network associated with the dynamics of the four opinions.

\section{A multi-group replicator model of opinion dynamics}

The model is comprised of three main elements: (i) individuals' opinions are affected by social influence; (ii) they further are sensitive to empirical information about the actual conflict between growth and environment; and (iii) policies may change the balance of opinions in some way, such as through influencing social interactions. We develop a multi-group replicator dynamics model extending basic replicator dynamics (Taylor and Jonker, 1978). The idea is close in spirit to Weisbuch et al. $(2002,2005)$ who posits the idea that agents tend to update their idea only if they interact with people within a certain threshold in their opinion spread. We approach this problem differently, though, namely by imposing four opinions and a network with local interactions between adjacent groups. Generalized to $n$ opinions, it takes the following form (for a derivation, see Appendix A):

$\dot{s}_{i}=s_{i}\left(f_{i}-\bar{f}_{i}\right)+\frac{1}{n} \sum_{j=1}^{n} s_{j}\left(\bar{f}_{j}-\bar{f}\right), i=1, \ldots, n$.

Here $\dot{s}_{i}\left(\equiv d s_{i} / d t\right)$ represents the change in the share of opinion $i$ in the population for the four opinions: Growth-at-all-cost (GC, or $i=1$ ), Green growth (GG, or $i=2$ ), Agrowth (AG, or $i=3$ ) and Degrowth (DG, or $i=4)$. The first term on the right-hand side of the equation represents the influence of group-specific interactions and the second of remaining interactions among individuals. The latter 
are the same for all opinions (needed to assure that shares add up to 1), causing the dynamics to be completely determined by differences in the group-specific interactions. The $\bar{f}_{i}$ denote group-specific average fitness, which deviate from global average fitness $\bar{f}$ in the standard replicator dynamics, as they are constructed taking into account the shares of each opinion $\left(s_{i}\right)$ and interaction networking intensities/psychological resistance $\left(w_{i}^{j}\right)$ with which individuals interact with others with a particular opinion:

$\overline{f_{i}}=\frac{w_{i}^{1} s_{1} f_{1}+\ldots+w_{i}^{i} s_{i} f_{i}+\ldots+w_{i}^{n} s_{n} f_{n}}{w_{i}^{1} s_{1}+\ldots+w_{i}^{i} s_{i}+\ldots+w_{i}^{n} s_{n}}$

The denominator in this equation assures that the fitness values $f_{i}$ are multiplied with weights $w_{j}^{i} s_{i}$ that always add up to one.

The symbol $w_{i}^{j}$ denotes networking intensity and psychological resistance. The first reflects the intensity of particular interactions in the network structure of interactions among individuals; and the second psychological resistance to other opinions. The double meaning suggests that with a low $w_{i}^{j}$ one is unlikely to change one's opinion as one communicates little with other opinions or has strong resistance against these.

The network of interactions is illustrated in Figure 1 for the case of local (group-internal) and adjacent-group interactions, consistent with the last row in Table 1. In the network of communication, individuals in GC and GG influence each other but those in AG and DG do not influence individuals in GC and vice versa. This structure reflects discounting of outlier opinions, where an outlier is defined relative to the opinion of each group. In other words, only opinions of adjacent groups directly influence individuals, not those in groups at further distance. This is consistent with empirical findings that environmental opinions tend to be segregated within like-minded communities or echo chambers (Williams et al., 2015).

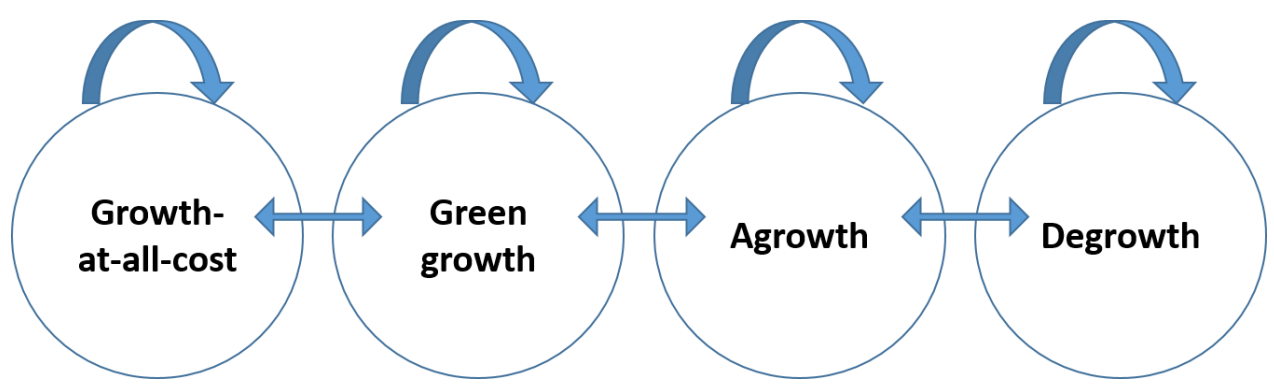

Figure 1. Social network of opinions based on in-group and adjacent interactions

We will consider two distinct matrices of networking intensities/psychological resistance $w_{j}^{i}$, as visualized in Figure 2. In case (a) these reflect that interactions among individuals are limited to own and adjacent groups, while opinions of individuals within the own group have a higher impact than those of individuals in adjacent groups, and opinions of individuals in groups further apart have no impact at all: $w_{i}^{i}>w_{i}^{j}>0 \forall i \neq j$ and $|i-j|=1$, and $w_{i}^{j}=0 \forall|i-j|>1$. The groups are ordered as GC-GG-AG-DG, as visually illustrated in Figure 1, with individuals in group GC interacting only with individuals in their own group and group GG, while individuals in group DG 
only interact with those in their own group and group AG. On the other hand, the "interior" groups GG and AG show a different interaction pattern, namely with members from the own-group and the two adjacent groups: i.e. individuals in group GG interact with those in groups GC, GG and AG, while individuals in group AG with GG, AG and DG. This network structure is consistent with Figure 1. For comparison, we also consider global interactions, that is, individuals being able to interact with individuals from any other, not only adjacent, group. This is illustrated by case (b) in Figure 2. Formally, networking intensities/psychological resistance are: $w_{i}^{i}>w_{i}^{j}>0 \forall|i-j| \geq 1$, thus, maintaining the logic that interaction between adjacent groups is stronger while also allowing for interaction with outlier opinions. Such global interactions might be considered as the outcome of applying public policies that stimulate interactions between individuals holding distant opinions. Concrete policy instruments in this respect are stimulating easy access to news media - or perhaps even free access for adolescents and young people up to a certain age, when opinions are formed. Other examples are communication of descriptive norms (Cialdini, 2007; Nyborg et al., 2016) and particular network interventions (Valente, 2012).

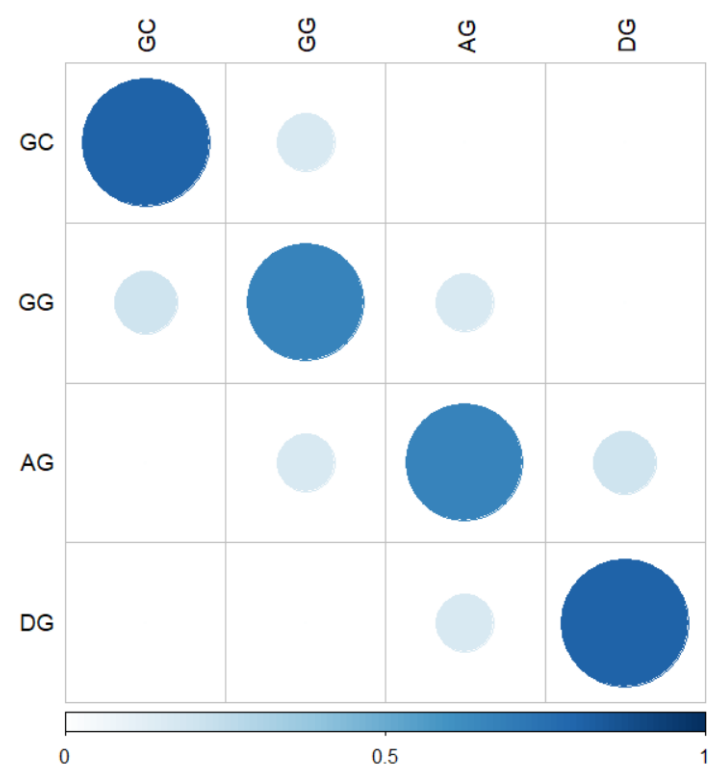

(a)

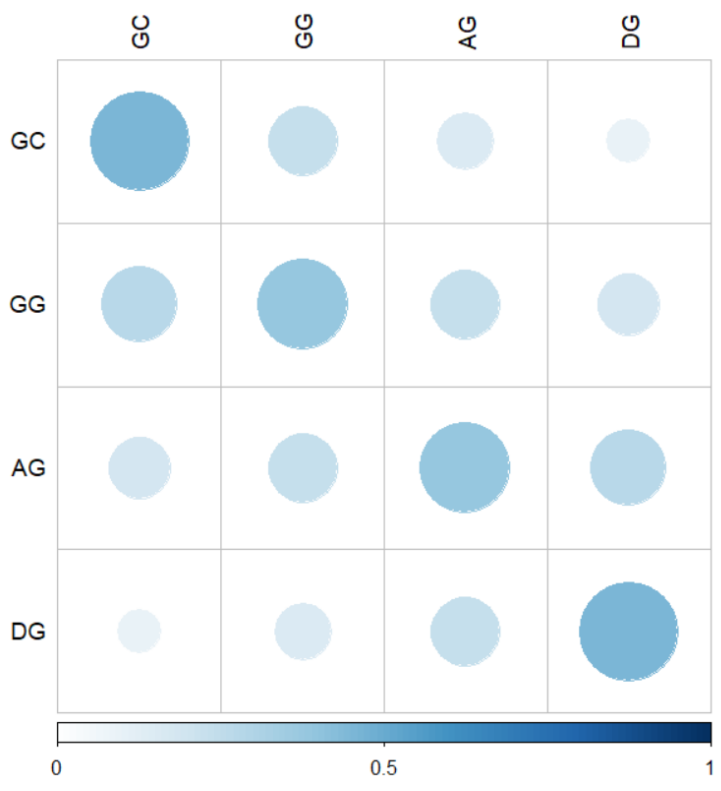

(b)

Figure 2. Illustration of social network structures assumed in the model, reflecting: (a) interactions among adjacent opinion groups, or (b) global interactions

Note: Colour and size of the circles illustrate the magnitude of the respective networking intensity/psychological resistance.

We further assume that people's environmental concern increases through direct and indirect experiences of environmental problems. To capture this, we include an index that expresses the development of environmental problems over time, such as climate change or biodiversity loss, under a pattern of continued economic growth. This might reflect what happens in reality or what science predicts will happen. A change in this index will have heterogeneous effects on the fitnesses of the distinct opinions (captured by the parameter $b_{i}$ - see Methods section for more details), in a way that 
worsening of environmental quality under growth will more positively affect the fitnesses of opinions more to the right in Figure 1. This is captured by the next equation.

$f_{i}=c+b_{i} E$

A change in this index will have heterogeneous effects on the fitnesses of the distinct opinions (captured by the parameter $b_{i}$ ), in a way that worsening of environmental quality under growth will more positively affect the fitnesses of opinions located to the right in Figure 1. We will assess the effects on opinion dynamics of both permanent and temporary environmental changes, as illustrated in Figure 3. This does not mean we assume this is the only possible scenario. However, the main alternatives, a constant $E$ over the entire period would translate into an exogenous and constant fitness, while a decreasing $E$ (improving environmental quality) would simply have the opposite effect of an increasing one. More details are provided in the Methods section.

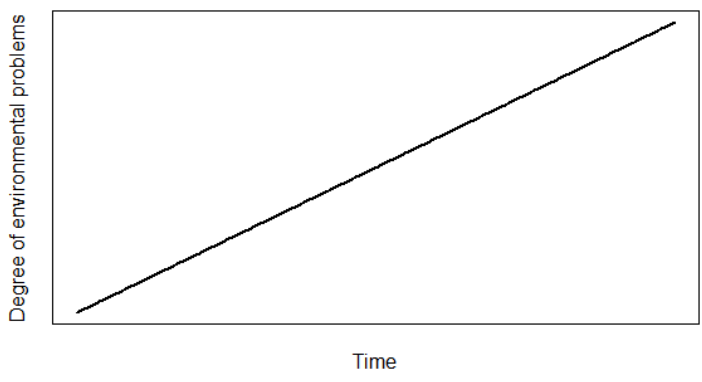

(a)

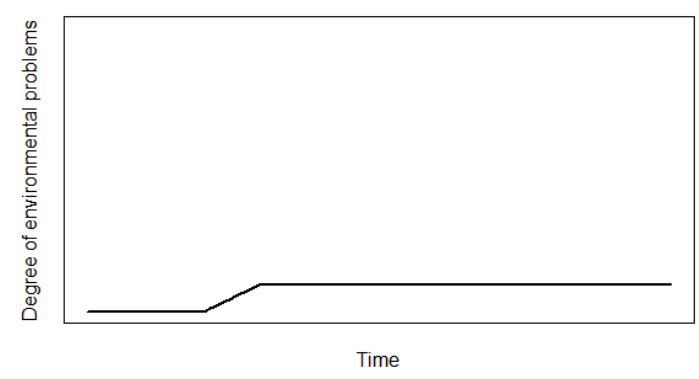

(b)

Figure 3. Scenarios of environmental change under continued economic growth: (a) permanent environmental deterioration, and (b) temporary environmental deterioration

\section{Results}

In the following we consider as a starting point a situation where opinion shares in the population are distributed as follows: $4 \%$ for GC, 59\% for GG, 21\% for AG and 16\% for DG. These values approximate empirical findings of Drews and van den Bergh (2016). The simulation period covers 500 time steps, assessed as sufficiently long for opinion shares to stabilize. Time steps might be interpreted as months or weeks, which translates into an effective period of roughly 10-40 years. The model was programmed in R software.

For adjacent interactions (consistent with Figure 2a) we assume $w_{i}^{i}=0.8, w_{i}^{j}=0.2 \forall i \neq$ $j$ and $|i-j|=1$, and $w_{i}^{j}=0 \forall|i-j|>1$. For global interactions (consistent with Figure 2b), we assume $w_{i}^{i}=0.8, w_{i}^{j}=0.2 \forall|i-j|=1, w_{i}^{j}=0.1$ for $|i-j|=2$ and $w_{i}^{j}=0.05$ for $|i-j|=3$, implying less intensive interactions among members of distinct groups than under adjacent interactions.

Opinion fitnesses are initially equal $\left(f_{i}=1 \forall\right.$ i), i.e. in equation 6 parameter $c=1$ (given that at time zero $E=0$ ). This can be motivated in two ways. First, different fitnesses arise immediately once environmental dynamics comes into play. Second, the assumption of equal fitness can be regarded as characterizing a stable starting point. Due to environmental dynamics as described by this equation, fitnesses alter as depicted in Figure $3 \mathrm{a}$ or $\mathrm{b}$, but to different degrees, depending on the values of $b_{i}$ for each opinion group, which we will vary, according to three cases as shown in Table 2 . The logic 
behind the choices is as follows: GC serves as reference value given that not all values need to change, as fitnesses only have meaning in terms of relative values; as explained in the previous section, DG is expected to respond strongly to environmental worsening, while AG may respond weakly or strongly, with the latter being more likely, and GG may also respond weakly or strongly, but with the first being more likely. This explains the distributions of parameter values for the three cases in the table. We adopt only values 0.1 and 0.5 to limit the number of combinations. We include equal values for some $b_{i}$ to test whether the joint survival of respective opinions in standard replicator dynamics upholds in our model under adjacent interactions. Considering a wider range of $b_{i}$ parameters with the same relative order will just rescale the time span within which one obtains the final outcomes: if $b_{i}$ values are very high (low) outcomes are reached rapidly (slowly).

Table 2. Scenarios for impact of environmental change on opinions through parameters $b_{i}$

\begin{tabular}{|l|l|l|l|l|}
\hline Cases & $\begin{array}{l}\boldsymbol{b}_{\mathbf{1}} \\
(\boldsymbol{G C})\end{array}$ & $\begin{array}{l}\boldsymbol{b}_{\mathbf{2}} \\
(\boldsymbol{G G})\end{array}$ & $\begin{array}{l}\boldsymbol{b}_{\mathbf{3}} \\
(\boldsymbol{A} \boldsymbol{G})\end{array}$ & $\begin{array}{l}\boldsymbol{b}_{\mathbf{4}} \\
(\boldsymbol{D} \boldsymbol{G})\end{array}$ \\
\hline$A$ & 0 & 0.1 & 0.1 & 0.5 \\
\hline$B$ & 0 & 0.1 & 0.5 & 0.5 \\
\hline$C$ & 0 & 0.5 & 0.5 & 0.5 \\
\hline
\end{tabular}

\section{Case A}

The top part of Figure 4 shows simulations for case $A$ in Table 2 with social interactions limited to adjacent groups. We observe the survival of two opinions, namely GG and DG. The left chart of the figure demonstrates how fitnesses of all four opinion groups change over time due to the environmental index increasing (equation 6), while the right chart illustrates the evolution of the shares of these opinions. While under standard replicator dynamics only one or multiple alternatives with (equal) highest fitness would survive, the figure shows that under multi-group evolution as in our model two opinions with distinct fitness values survive, namely GG next to the best-fitness opinion DG. The reason is that the latter is buffered from the effect of a growing share of opinion DG by the existence of an 'in-between' opinion AG in the network structure. In other words, local selection effects assure survival of more opinions, as opposed to replicator dynamics where all selection is global in nature so that no such buffering is possible. This is illustrated in the second top panel in Figure 4, where, consistent with Figure 2b, we allow for interaction between opinion groups being further away from each other (global social interactions). In this case, DG is the only surviving opinion in the long run.

We also undertook simulations for the same case $A$, but with environmental change occurring only in a limited time window (periods 101-150, as illustrated in Figure 3b). The results of this, shown in Figure B1 (in Appendix B), show that the stable outcomes remain the same (i.e. two and one opinions dominate in the long run, respectively), highlighting that very small fitness differences due to temporary environmental change are sufficient to achieve the same patterns.

\section{Case B}

The middle part of Figure 4 contains the results of simulations for case $B$ in Table 2. If social interactions are limited to adjacent groups, only opinion GG disappears in the long run while the adjacent opinion AG dominates, and GC survives at a level close to its initial share. Here, GC is buffered in a negative way by the extinction of adjacent opinion GG, cutting any indirect interactions 
with groups exhibiting higher fitness (AG in this case). The small increases in the shares of GC and DG (between periods 101 and 150) is due to the second term in equation 5, which distributes a small part of the disappearing GG opinions to the two extreme opinion groups. Figure 4 indicates that if, under case B, communication between groups is global, only AG and DG opinions survive.

If we now again consider environmental change to be restricted to a limited time interval (consistent with Figure 3b), our results do not change much (see Figure B1 in Appendix B).

\section{Case $C$}

The bottom part of Figure 4 shows simulations for case $C$ in Table 2, with adjacent and global interactions among opinion groups under permanent environmental change (results under temporary environmental change are in the bottom part of Figure B1 in Appendix B). Visual inspection suggests that the results are identical, but in fact they differ slightly. The reason for similarity is that the outcomes are dominated by the assumption of equal impacts of environmental change on fitnesses.

We further experimented with alternative networking intensities/psychological resistance for local interactions (Figure 2a), namely shifting them from the interaction inside the group to the interaction with members of the group(s) adjacent to it. In particular, we tried extreme values $w_{i}^{i}=$ 0.51 and $w_{i}^{j}=0.49$, an option with the most intense between-group interaction given the condition stated in Appendix A that people interact more with those holding a similar opinion, $\left(w_{i}^{i}>w_{i}^{j}>\right.$ $0 \forall i \neq j$ and $|i-j|=1$ ). In this case, our model produces the same (qualitative) patterns of opinion distribution as reached under adjacent interactions with networking intensity/psychological resistance values as in the previously presented results (Figure 4). That is, the same opinions survive, and even their rank stays the same, demonstrating robustness of our results to variation in parameter values. Results of this exercise are shown in Figure B2 (Appendix B).

As an additional robustness test, we examine what happens if psychological resistance is higher, in line with our earlier discussion on this. To achieve this, we reduce the variable "networking intensities/psychological resistance" for adjacent groups by half and repeat the experiments described for cases A, B and C. Results, reported in Figure B3 (Appendix B), alter very little and remain qualitatively the same. Due to higher psychological resistance the shares of opinions change more slowly. For instance, in case B, opinion AG reaches an $80 \%$ share not after 150 but 200 periods. 

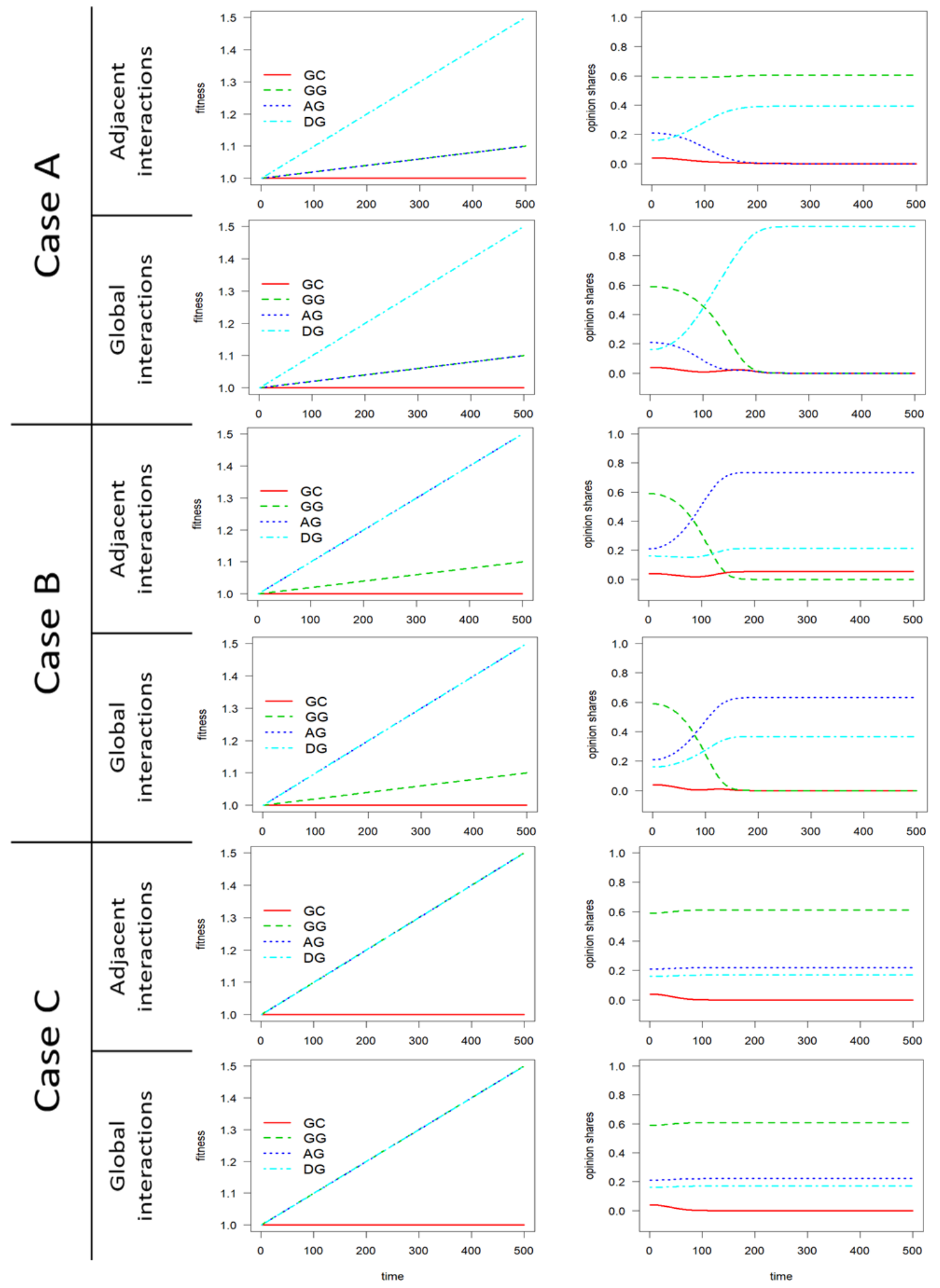

Figure 4. Dynamics of opinion fitnesses and shares over time under permanent environmental change 


\section{Extending the model with policy feedback}

So far we assumed the degree of environmental problems $(E)$ under continued growth to be exogenous. Here we examine the robustness of the previous results under an additional feedback, namely that opinions affect policy, in turn the environment, and then back to opinions (Figure 5).

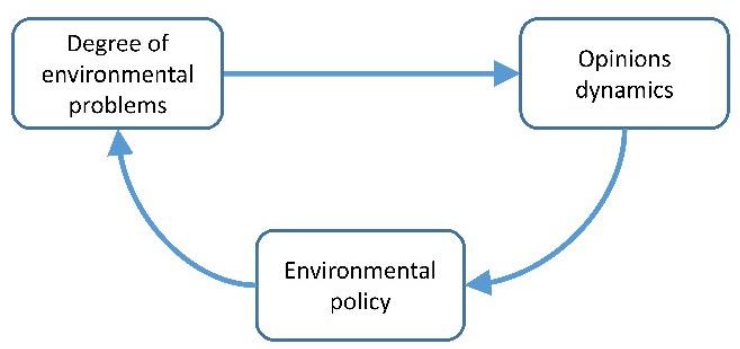

Figure 5. Adding policy feedback

The main challenge here is translating opinions to policy. Since important (global) environmental problems are cumulative in nature - i.e. pollution in the past affects the degree of current environmental problems - environmental policy can only affect the change of such problems $\left(\Delta E_{t}\right)$. We calculate this change by weighting the policy impact of each of the four opinions $\left(\gamma_{i}\right)$ in each period by their respective shares $\left(s_{i}\right)$. Since we assume a linear shape of impact curves for $E_{t}$, these $\gamma_{i}$ take on constant values. In formal terms we have:

$$
E_{t}=E_{t-1}+\Delta E_{t}=E_{t-1}+s_{t, G C} \gamma_{G C}+s_{t, G G} \gamma_{G G}+s_{t, A G} \gamma_{A G}+s_{t, D G} \gamma_{D G}
$$

We assume the following inequality $\gamma_{G C}>\gamma_{G G}>\gamma_{A G}=\gamma_{D G}$ to hold, as illustrated in Figure 6. It means that if everyone has the GG opinion then the middle curve applies (i.e. identical to Figure $3 \mathrm{a}$ of the basic model); if all opinions are GC then the top curve is relevant, associated with weaker environmental policy and more focus on pure economic growth, which leads to a faster increase of environmental problems; and if everyone supports DG or AG the bottom line applies, based on the idea that the implied policies slow down environmental problems. What equation 7 does is at each time weighting the associated values on the three curves with the shares of the associated opinions to derive the environmental change. This can be seen as representing an underlying democratic process, such as a governing coalition government with participating parties finding a middle ground between the prevailing opinions and translating this into policy. 


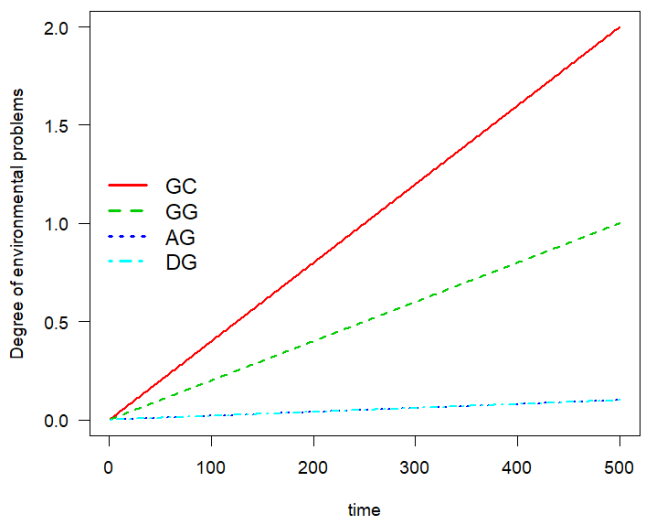

Figure 6. Degree of environmental problems depending on the dominant opinion

Including this additional feedback, we run the extended model and report the results in Figure B4a (in Appendix B). They are close to the ones obtained with the basic model (Figure 4), demonstrating robustness of our previous findings. We also considered an alternative political-democratic case where policy is determined by the opinion with the highest share rather than by an average of opinions as in equation 7. This does not produce significantly different results (Figure B4b in Appendix B). Our analysis here merely illustrates that our model is capable of addressing this issue, and that its inclusion does not much affect the outcomes. This line of research could be further developed by incorporating knowledge from political sciences about how the distribution of political opinions precisely affects environmental policy stringency. But since this distribution will take some form in between the extremes examined here, it is not to be expected to alter our findings.

\section{Interpretations and conclusion}

The results of the simulations are summarized in Table 3. Three main insights can be derived from it. First, adjacent social interactions among groups - arguably the more realistic assumption for the current opinion dynamics on growth-versus-environment - generate distinct results from global interactions in terms of long term survival of growth-at-all-cost and green growth opinions (green coloured text in Table 3) but not of agrowth and degrowth opinions. Second, equal impacts of environmental change on opinions as shown in Table 2 (e.g., for green growth and agrowth in case A) do not always translate in joint survival of associated opinions. Third, the three cases address uncertainty about how environmental change affects opinion fitnesses, suggesting overall that growth-at-all-cost has the highest likelihood of disappearing, followed by green growth and agrowth, while degrowth always survives. Moreover, these qualitative outcomes are robust for adjacent versus global interactions, except with respect to green growth under case A and growth-at-all-cost under case B. Clearly, under local or adjacent interactions more diversity of opinions survives due to the extinction of certain opinions creating a barrier which prevents communication between individuals holding very distinct opinions.

The results also provide information about the final distribution of opinion shares, which has relevance as we set the initial distribution in accordance with recent public opinion research. Under adjacent interactions, the share of green growth is highest in cases $A$ and $C$, while in case $B$ agrowth dominates. If social interactions are global, degrowth dominates in case $A$, agrowth in case $B$ and green growth in case $C$. Table 3 reports the ultimate shares as well as their averages for all three cases. Under the more realistic case of adjacent interactions, the three opinions green growth, agrowth and 
degrowth perform rather similar in terms of survival, though they have distinct average opinion shares. Under global interactions, green growth performs much worse, agrowth almost the same and degrowth better.

Generally, we find that under adjacent interactions among opinion groups, ultimate outcomes are characterized by diversity of opinions, i.e. a lack of consensus. These results suggest that the growth-vs-environment debate is likely to continue for a long time into the future. However, our findings also suggest that under worsening environmental conditions AG and DG become politically more feasible as people shift away from GG to these two opinion groups. If one were to implement policies that foster interactions beyond adjacent groups, i.e. global interactions, then an evolution towards consensus would be more likely. Such consensus might contribute to achieving critical support for sustainability policies. We extended the basic model with feedback from opinions to policy and to environmental change. It shows the robustness of our findings, as well as that our model is capable of addressing this issue.

As this is the first study of its kind, there is ample room for further research. Next to the impact of environmental change on opinions, one could account for the effect of low growth or a recession on opinions. We have not considered this case as this particular causality has not been observed in reality, which also means that there is no relevant empirical data to undertake model calibration for analysing such a case. Another, somewhat related case is an exogenous economic crisis that reduces concern about environmental problems (Scruggs and Benegal, 2012), which might be interpreted as potentially shifting opinions from AG and DG to GC and GG. Next, the assumptions of linearity in Figure 3 and equation 6 might be replaced by nonlinear relationships - whether concave or convex - based on empirical evidence about how quickly people have changed their opinions in response to concrete environmental changes. On the other hand, these assumptions, including the scaling of the parameters $b_{i}$, are not very restrictive as they do not affect the final stable distribution of opinions but only the speed of opinion dynamics and, thus, the time at which stability is achieved. Another line of research is to develop an agent-based model (ABM) of opinion dynamics to address diversity of individuals in a more sophisticated manner. In tandem, additional empirical or experimental research would be required to support the larger number of parameter values in such an ABM approach. Finally, feedback from opinion to policy to environmental-change could be elaborated by including relevant insights from political sciences about how exactly the distribution of political opinions affects environmental policy stringency.

Table 3. Summary of opinion survival for adjacent/global interactions

\begin{tabular}{|l|c|c|c|c|}
\hline Case & $\begin{array}{c}\text { Growth-at-all-cost } \\
(G C)\end{array}$ & $\begin{array}{c}\text { Green growth } \\
(G G)\end{array}$ & $\begin{array}{c}\text { Agrowth } \\
(A G)\end{array}$ & $\begin{array}{c}\text { Degrowth } \\
\text { (DG) }\end{array}$ \\
\hline A & disappears/disappears & survives/disappears & disappears/disappears & survives/survives \\
& $(0.00 / 0.00)$ & $(0.61 / 0.00)$ & $(0.00 / 0.00)$ & $(0.39 / 1.00)$ \\
\hline$B$ & survives/disappears & disappears/disappears & survives/survives & survives/survives \\
& $(0.05 / 0.00)$ & $(0.00 / 0.00)$ & $(0.74 / 0.63)$ & $(0.21 / 0.37)$ \\
\hline C & disappears/disappears & survives/survives & survives/survives & survives/survives \\
& $(0.00 / 0.00)$ & $(0.61 / 0.61)$ & $(0.22 / 0.22)$ & $(0.17 / 0.17)$ \\
\hline Average & $(0.02 / 0.00)$ & $(0.40 / 0.20)$ & $(0.32 / 0.28)$ & $(0.26 / 0.51)$ \\
values & & & & \\
\hline
\end{tabular}


Note: Ultimate opinion shares for adjacent/global interactions are reported in parentheses. Green colour denotes combinations with distinct survival outcomes under adjacent and global interactions.

\section{Appendix A. The basic model}

We develop a multi-group replicator dynamics model. It extends the basic replicator dynamics (Taylor and Jonker, 1978) for $n$ strategies (in our case opinions), which describes a single group of individuals, that is, global interactions among individuals:

$\dot{s}_{i}=s_{i}\left(f_{i}-\bar{f}\right), i=1, \ldots, n$.

Here $\dot{s}_{i}\left(\equiv d s_{i} / d t\right)$ represents the change in the share of opinion $i$ in the population. For a detailed treatment and extensions of this model, see Nowak (2006). Average fitness is defined as the weighted sum of the fitnesses of each opinion $f_{i}$ :

$\bar{f}=\sum_{i=1}^{n} s_{i} f_{i}$

The aggregate character of replicator dynamics allows multiple interpretations, notably learning, imitation and conformism. Either is relevant to our application as individuals may learn by studying information about the relationship between growth and the environment, or copy the opinions of others who they trust, or be conformist, i.e. follow the majority opinion.

To more precisely address assortment and conformism, we are interested in extending the simple replicator model to a population with subgroups. This is not to be confused with multipopulation replicator dynamics, a special case of multi-population games, which describes interactions among groups of individuals with distinct roles, such as police and hooligans, each with specific strategy sets (Bennet, 1980; Kanazawa et al., 2003). Instead, our approach is closer to what is known as replicator dynamics with population structure or assortment (see e.g., Fatas-Villafranca et al., 2011 and Almudi et al., 2017), which leads to departures from random matching as in the homogenous population replicator case (van Veelen, 2011). To allow for describing particular interactions between groups in the population, each consisting of individuals with the same option, we rewrite equation 1 as:

$\dot{s}_{i}=s_{i}\left(f_{i}-\bar{f}_{i}\right)+s_{i}\left(\bar{f}_{i}-\bar{f}\right), i=1, \ldots, n$.

The first part reflects the influence of group-specific interactions and the second of global interactions. Here $\bar{f}_{i}$ denotes group-specific average fitness, which deviates from global average fitness $\bar{f}$. They are defined as:

$\bar{f}_{i}=\frac{w_{i}^{1} s_{1} f_{1}+\ldots+w_{i}^{i} s_{i} f_{i}+\ldots+w_{i}^{n} s_{n} f_{n}}{w_{i}^{1} s_{1}+\ldots+w_{i}^{i} s_{i}+\ldots+w_{i}^{n} s_{n}}$

where $w_{i}^{j}$ denotes the networking intensity/psychological resistance of the contributing fitness of individuals in group $j$ to the effective average fitness (i.e. which influences change of opinions) of individuals in group $i$ (with $0 \leq w_{i}^{j} \leq 1$ ). If $w_{i}^{j}=1 \forall i, j$, then $\bar{f}_{i}=f_{i} \forall i$ causing equation 3 to 
simplify to 1 . In other words, the $w_{i}^{j}$ represent the network structure of interactions among individuals. The details of this were discussed in the main text.

The expression in equation 4 demonstrate that the $w_{j}^{i} s_{i}$ effectively function as networking intensities/psychological resistance. But while $s_{i}$ are endogenous and reflect the variable number of individuals in adjacent groups that may influence each other, $w_{j}^{i}$ are exogenous and capture more stable personality or behavioural factors, such as openness to other opinions, docility or conformity, and even curiosity. These fitness specifications may thus be seen to capture average susceptibility of individuals in a group to other opinions, even though in reality there may be diversity: for instance, some individuals are strongly convinced about their opinion or not influenced by individuals or opinions from other groups, while others may have doubts or be in contact with outgroup-individuals. The right hand side of equation 4 contains a denominator to assure that the fitness values are multiplied with networking intensities/psychological resistance $\frac{w_{i}^{i} s_{i}}{w_{i}^{1} s_{i}+\ldots+w_{i}^{i} s_{i}+\ldots+w_{i}^{n} s_{n}}$. Note that these weights add up to one if summed over $i$, (i.e. one does not need to impose the separate condition $\left.\sum_{i=1}^{n} w_{i}^{j}=1\right)$ and are replaced by a zero value if $w_{i}^{i} s_{i}$ and the denominator are both zero (to avoid the problem of dividing by zero).

Equation 4 reflects potential asymmetry of effects of adjacent groups. This can work three ways. First, the value of the fitness parameter $f_{i}$ matters, and it can differ between adjacent groups, so that one group has a stronger intrinsic influence on adjacent groups than another. Second, the size of the group matters, which captures the fact that a larger group of individuals with a certain opinion has, ceteris paribus, more potential to influence the opinions of individuals in an adjacent group than a smaller group. One might also use the networking intensities/psychological resistance (or the fitnesses) to capture individual differences in terms of commitment to their position (Xie et al., 2011).

In equation 3 the first term $s_{i}\left(f_{i}-\bar{f}_{i}\right)$ represents direct group interactions, but there still is a second term, namely $s_{i}\left(\bar{f}_{i}-\bar{f}\right)$. The latter can be interpreted as remaining global interactions. We would now like to cancel any effects of the latter, as we want to model a situation that allows for only local effects or interactions between adjacent groups. The way to accomplish this is revising equation 3 , under the constraint that the changes described in shares sum up to zero (i.e. the shares sum up to one). We do this by calculating the sum of all the second terms across the four groups, leading to what we might call the global effect $g=\sum_{j=1}^{n} s_{j}\left(\bar{f}_{j}-\bar{f}\right)$, and then replacing the second term in equation $3 \mathrm{by} \mathrm{g} / n$. This will result in an equal (though not constant) global effect for each group, which summed is equal to $n \cdot g / n=g$. In other words, the sum of the shares does not alter, while the differences between the dynamics of the shares is determined now by the first term, that is, groupinternal and group-adjacent opinion influences. Equation 2 then is replaced by the following adapted replicator equation (generalized for $n$ opinions):

$\dot{s}_{i}=s_{i}\left(f_{i}-\bar{f}_{i}\right)+\frac{1}{n} \sum_{j=1}^{n} s_{j}\left(\bar{f}_{j}-\bar{f}\right), i=1, \ldots, n$.

People's environmental concern increases through direct and indirect experiences of environmental problems. To capture this, we include an exogenous index $E$ expressing the development of environmental pressure or problems over time, such as climate change or biodiversity loss, under an assumed pattern of continued economic growth. A rise in this index denotes a deterioration of the environment, which will have heterogeneous effects on fitnesses of distinct 
opinions $f_{i}$. In particular, the opinion fitnesses are defined by an exogenous, constant part $c$ and a variable, endogenous part, as follows (assuming that the initial value of index $E$ is zero):

$f_{i}=c+b_{i} E$

With respect to sensitivities to real-world changes, we make the following assumptions. GC ignores the environment. Although one might think this view is extreme, Drews and van den Bergh (2016) find that a small proportion of individuals falls into this category. This study further suggests that on the other end of the spectrum, DG shows the highest sensitivity to environmental change, indicating that support of degrowth is significantly associated with environmental values. The two remaining groups GG and AG fall in-between. Formally, these assumptions mean that $0=b_{1} \leq b_{2} \leq b_{3} \leq b_{4}$ to reflect that worsening of environmental quality under growth will generally more positively affect the fitnesses of opinions more to the right in Figure 1. This might be seen as a special case of a more general phenomenon that people pay more attention to those issues that correspond to their worldview. Note that since only relative values of fitnesses matter, we can set $b_{1}=0$. As for the environmental quality component $E$, starting at a value zero (no impact on fitnesses), we will consider the case that over time it deteriorates, captured by a linear increase over the entire period (permanent environmental change, such as global warming), or only for a short time interval (temporary environmental change, such as ozone depletion), as illustrated in Figure 3. This allows us to assess the differential effects of permanent and temporary environmental changes on opinions. Note that a constant $E$ over the entire period considered would translate into an exogenous and constant fitness, while a decreasing $E$ (improving environmental quality) would have the opposite effect of an increasing one. For these reasons, and as these cases are less relevant, we do not consider them. 
Appendix B. Results from sensitivity analyses and policy feedback
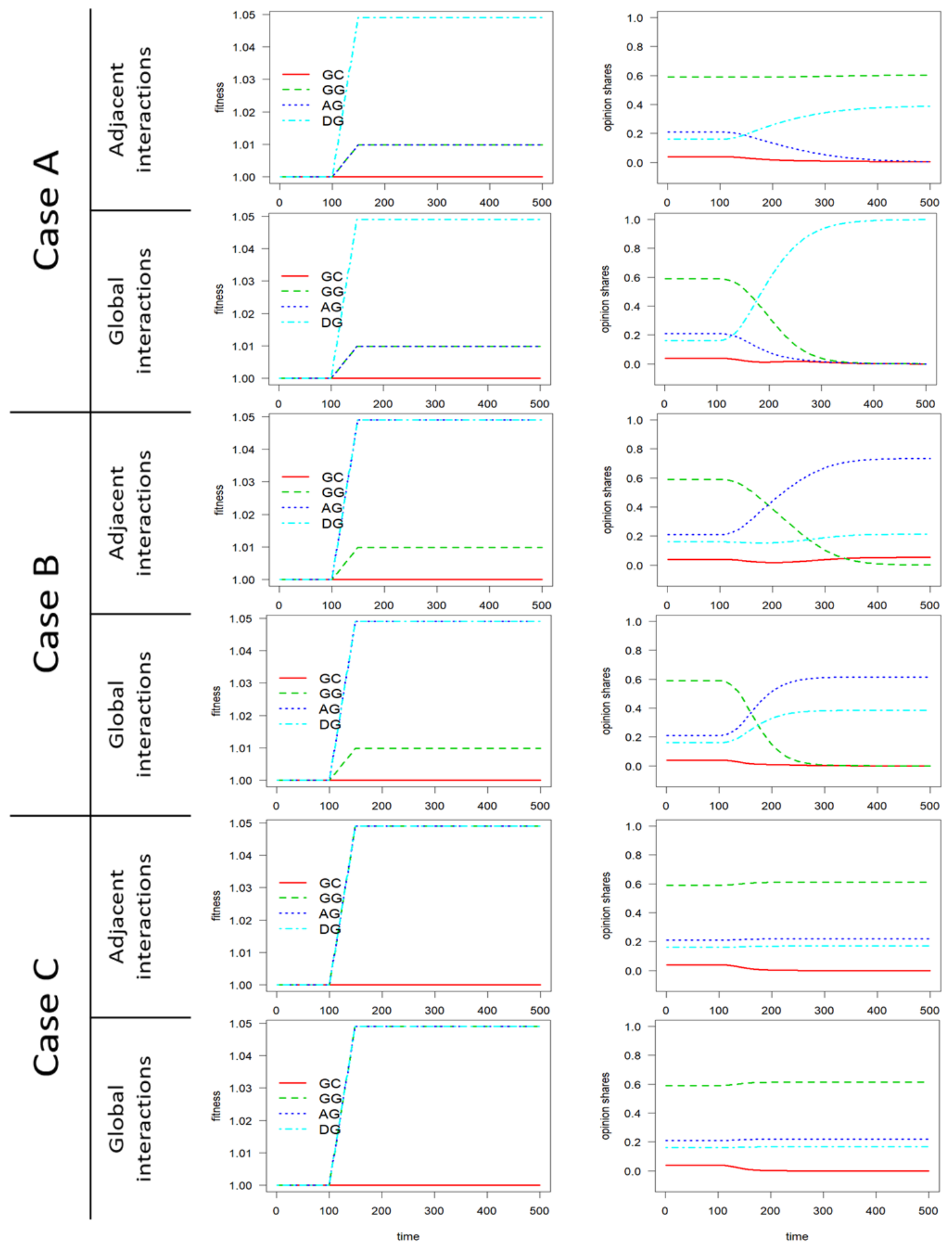

Figure B1. Dynamics of opinion fitnesses and shares over time under temporary environmental change 


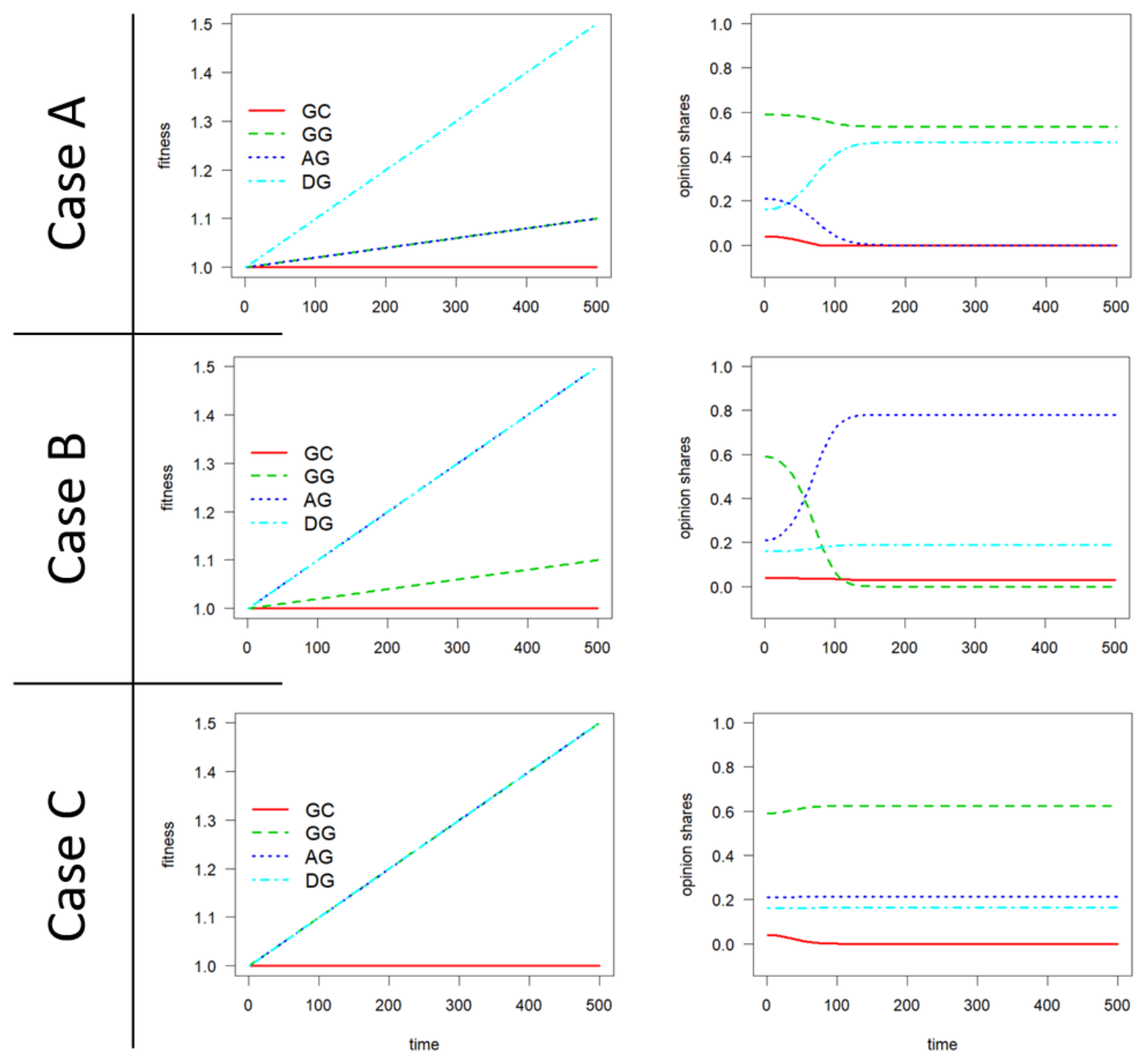

Figure B2. Dynamics of opinion fitnesses and shares over time under permanent environmental change and alternative networking intensities/psychological resistance for local interactions $\left(w_{i}^{i}=\right.$ 0.51 and $w_{i}^{j}=0.49$, where $|i-j|=1$ ) 

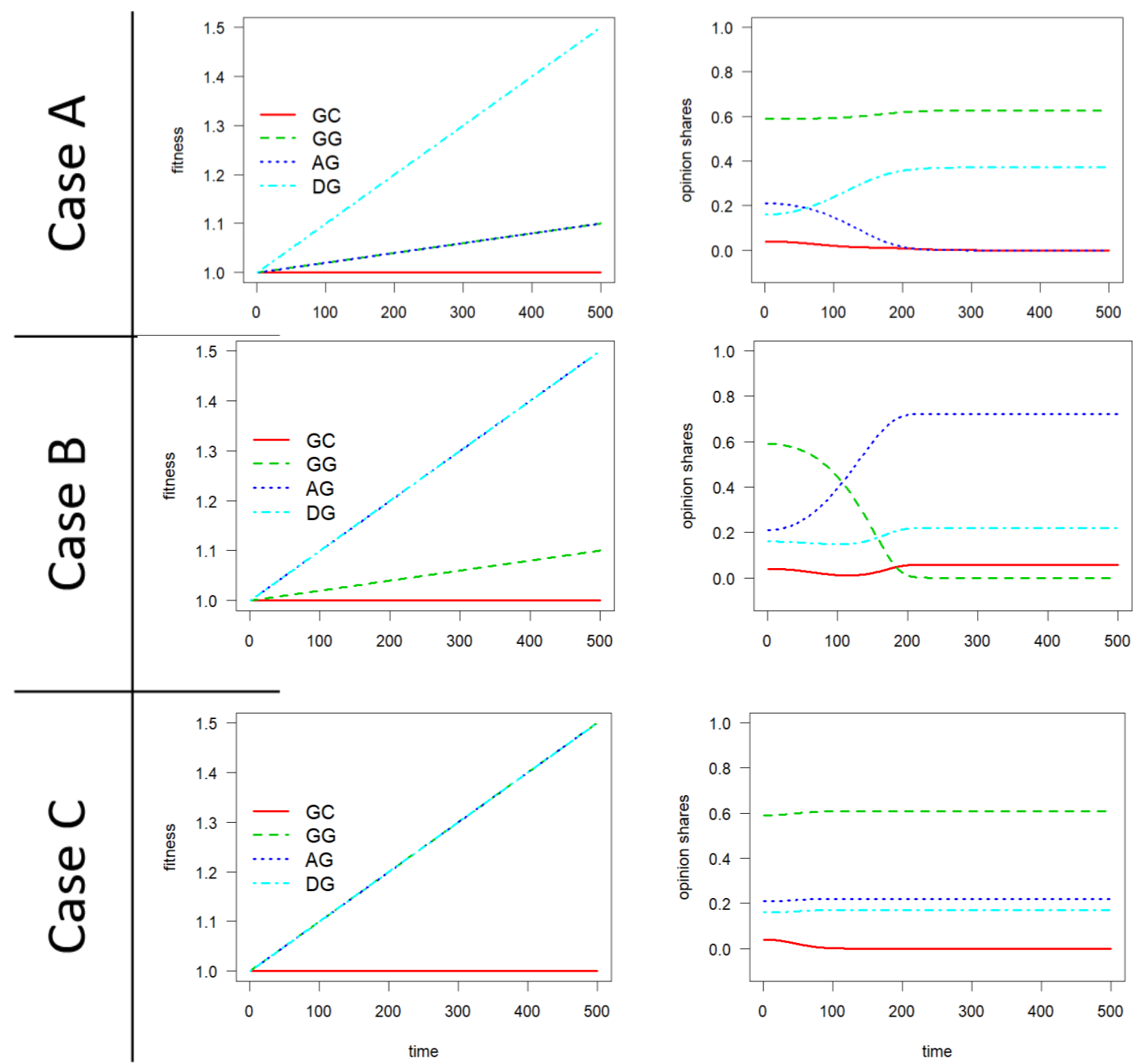

Figure B3. Dynamics of opinion fitnesses and shares over time under permanent environmental change and higher psychological resistance $\left(w_{i}^{i}=0.8\right.$ and $w_{i}^{j}=0.1$, where $\left.|i-j|=1\right)$ 


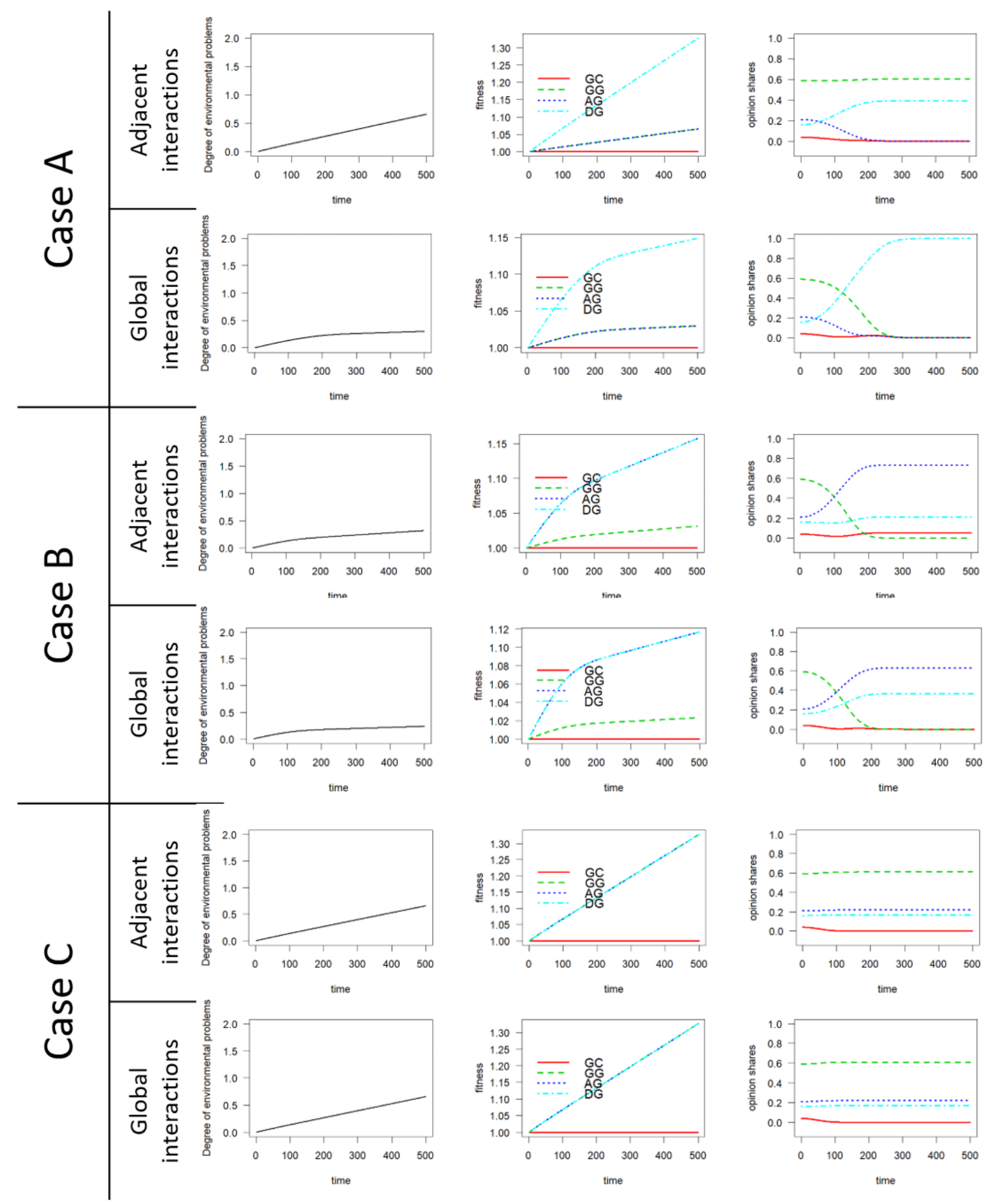

Figure B4a. Dynamics of opinion fitnesses and shares over time under policy feedback (equation 7) 


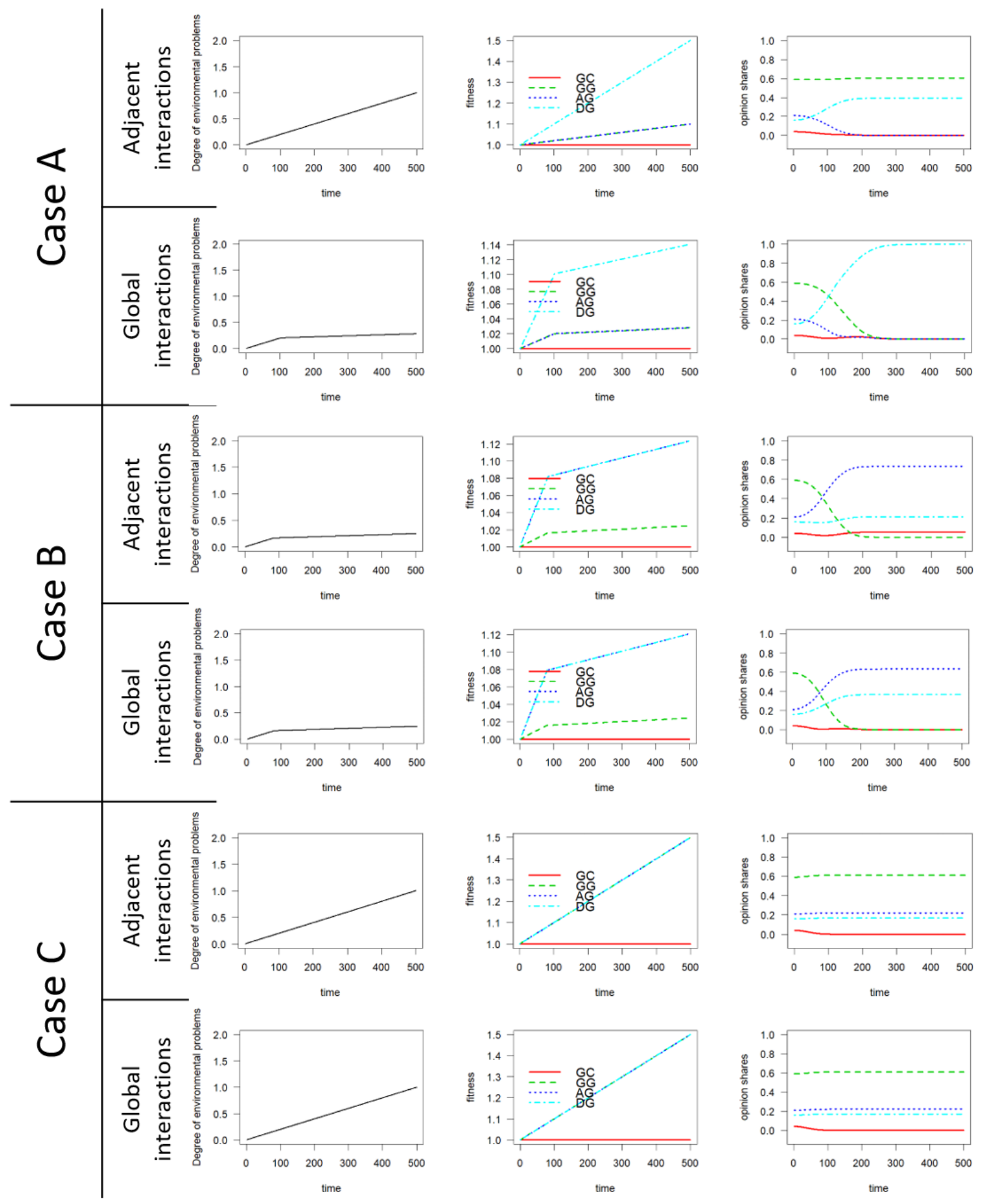

Figure B4b. Dynamics of opinion fitnesses and shares over time under policy feedback (majority dominance) 
Acknowledgements: This study has received funding through an ERC Advanced Grant from the European Research Council (ERC) under the European Union's Horizon 2020 research and innovation programme (grant agreement $n^{\circ}$ 741087). Ivan Savin also acknowledges RFBR grant № 18-010-01190. We thank Franziska Klein and Filippos Exadaktylos for useful comments.

\section{References}

Almudi, I., Fatas-Villafranca, F., and J. Potts (2017). Utopia competition: a new approach to the micro-foundations of sustainability transitions. Journal of Bioeconomics 19:165-185.

Anderson, B., T. Böhmelt and H. Ward (2017). Public opinion and environmental policy output: a cross-national analysis of energy policies in Europe. Environmental Research Letters 12(11), 114011.

Arrow, K.J., Bolin, B., Costanza, R., Dasgupta, P., Folke, C., Holling, C.S., et al (1995). Economic growth, carrying capacity, and the environment. Science 268(April): 520-521.

Beckerman, W. (1976). In Defence of Economic Growth. Johathan Cape, London.

Bennett, P.G. (1980). Using hypergames to model difficult social issues: an approach to the case of soccer hooliganism. Journal of the Operational Research Society 31(7): 621-635.

Capstick, S., Whitmarsh, L., Poortinga, W., Pidgeon, N., and Upham, P. (2015). International trends in public perceptions of climate change over the past quarter century. Wiley Interdisciplinary Reviews: Climate Change 6, 35-61.

Castellano C. Fortunato S. and Loreto V. (2009). Statistical physics of social dynamics. Reviews of Modern Physics 81(April-June): 591-646.

Cialdini, R. (2007). Descriptive social norms as underappreciated sources of social control. Psychometrika 72(2): 263-268.

Daly, H. E. (1977). Steady-state Economics. W.H. Freeman, San Francisco.

Demski, C., Capstick, S., Pidgeon, N., Sposato, R.G., and Spence, A. (2017). Experience of extreme weather affects climate change mitigation and adaptation responses. Climatic Change 140, 149164.

Drews, S., and J.C.J.M. van den Bergh (2016). Public views on economic growth, the environment and prosperity: Results of a questionnaire survey. Global Environmental Change 39: 1-14.

Drews, S. en J.C.J.M. van den Bergh (2017). Scientists' views on economic growth versus the environment: A questionnaire survey among economists and non-economists. Global Environmental Change, 46: 88-103.

Drews S., M. Antal and J. van den Bergh (2018). Challenges in assessing public opinion on economic growth versus environment: Considering European and US data. Ecological Economics 146: 265272.

Fatas-Villafranca, F., D. Saura and F.J. Vazquez (2011). A dynamic model of public opinion formation. Journal of Public Economic Theory 13(3): 417-441.

Gargiulo F., and J.J. Ramasco (2012). Influence of opinion dynamics on the evolution of games. PLoS ONE 7(11): e48916.

Galbraith, J.K. (1958). The Affluent Society. Houghton Mifflin Company, Boston.

Higgs, K. (2014). Collision Course: Endless Growth on a Finite Planet. The MIT Press, Cambridge, Mass.

Hirsch, F. (1976). Social Limits to Growth. Harvard University Press, Cambridge, MA.

Howe, L.C., and J.A. Krosnick (2017). Attitude Strength. Annual Review of Psychology 68: 327-351. Jackson, T. (2009). Prosperity Without Growth-Economics for a Finite Planet. Earthscan, London. 
Janssen M., de Vries B. (1998). The battle of perspectives: a multi-agent model with adaptive responses to climate change. Ecological Economics 26: 43-66.

Johnston, C.D., and J. Wronski (2013). Personality dispositions and political preferences across hard and easy issues. Political Psychology 36(1): 35-53.

Johnson, D.R. (2017). Bridging the political divide: Highlighting explanatory power mitigates biased evaluation of climate arguments. Journal of Environmental Psychology 51: 248-255.

Kahan, Dan M., Ellen Peters, Maggie Wittlin, Paul Slovic, Lisa Larrimore Ouellette, Donald Braman, and Gregory Mandel. 2012. "The Polarizing Impact of Science Literacy and Numeracy on Perceived Climate Change Risks." Nature Climate Change 2(5): 732-735.

Kahneman, D., Krueger, A., Schkade, D., Schwarz, N. and Stone, A. (2004). Toward national wellbeing accounts. American Economic Review, Papers and Proceedings 94: 429-434.

Kanazawa, T., T. Ushio, and T. Yamasaki (2003). Replicator dynamics of evolutionary hypergames. In: Proceedings of 2003 IEEE International Conference on Systems, Man, and Cybernetics, pp. $3828-3833$.

Lorenz, J., H. Rauhut, F. Schweitzer, and D. Helbing (2011). How social influence can undermine the wisdom of crowd effect. Proceedings of the National Academy of Sciences 108(22): 90209025.

Maddox, J. (1972). The Doomsday Syndrome. Macmillan, London.

Meadows, D.H., Meadows, D.L., Randers, J. \& Behrens, W.W. (1972). The Limits to Growth. Universe Books, New York.

Mishan, E. J. (1967). The Cost of' Economic Growth. Staples Press, London.Nyborg, K. et al. (2016). Social norms as solutions. Science 354(6308): 42-43.

Nowak, M. A. (2006). Evolutionary Dynamics. Exploring the Equations of Life. Harvard University Press, Cambridge, MA.

Porter, E. (2015). Imagining a world without growth. The New York Times, 1 December 2015. Retrieved from: https://www.nytimes.com/2015/12/02/business/economy/imagining-a-worldwithout-growth.html

Sandbach, F. (1978). The rise and fall of the limits to growth debate. Social Studies of Science 8: 495520.

Schmelzer, M. (2015). The growth paradigm: History, hegemony, and the contested making of economic growthmanship. Ecological Economics 118: 262-271.

Shalizi C.R., and A.C. Thomas (2011). Homophily and contagion are generically confounded in observational social network studies. Sociological Methods and Research 40(2): 211-239.

Scruggs, L., and S. Benegal (2012). Declining public concern about climate change: Can we blame the great recession? Global Environmental Change 22(2): 505-515

Shi, J., V.H.M. Visschers, M. Siegrist and J. Arvai (2016). Knowledge as a driver of public perceptions about climate change reassessed. Nature Climate Change 6(4): 759-762. |

Simon, J. (1981). The Ultimate Resource (2nd ed., 1996). Princeton University Press, Princeton.

Sîrbu, A., V. Loreto, V.D.P. Servedio, and F. Tria (2017). Opinion dynamics: Models, extensions and external effects. Chapter 17 in: V. Loreto et al. (eds.), Participatory Sensing, Opinions and Collective Awareness, Understanding Complex Systems, Springer, Switzerland.

Taylor, P., and L. Jonker (1978). Evolutionary stable strategies and game dynamics. Mathematical Biosciences 40: 145-156. 
Tjernström, E., and T.Tietenberg (2008). Do differences in attitudes explain differences in national climate change policies? Ecological Economics 65 (2): 315-324.

Tur, E.M., P. Zeppini and K. Frenken (2018). Diffusion with social reinforcement: The role of individual preferences. Physical Review E 97 (2), 022302.

Turner, G., and Alexander, C. (2014). Limits to Growth was right. New research shows we are nearing collapse. The Guardian, 2 September 2014. Retrieved from: https://www.theguardian.com/commentisfree/2014/sep/02/limits-to-growth-was-right-newresearch-shows-were-nearing-collapse

Valente T.W. (2012). Network interventions. Science 337(6090): 49-53.

van den Bergh, J.C.J.M. (2017). A third option for climate policy within potential limits to growth. Nature Climate Change 7(2): 107-112.

van Veelen, M. (2011). The replicator dynamics with n players and population structure. Journal of Theoretical Biology 276(1): 78-85.

Vazquez, F., P.L. Krapivsky, and S. Redner (2003). Constrained opinion dynamics: Freezing and slow evolution. Journal of Physics A36: L61.

Victor, P.A. (2008). Managing without Growth. Slower by Design, Not Disaster. Edward Elgar. Cheltenham, U.K. and Northampton, MA, USA.

Weisbuch G., G. Deffuant, F. Amblard and J.P. Nadal (2002). Meet, discuss, and segregate! Complexity 7(3): 55-63.

Weisbuch G., G. Deffuant and F. Amblard (2005). Persuasion dynamics. Physica A 353: 555-575.

Williams, H.T.P., J.R. McMurray, T. Kurz and F.H. Lambert (2015). Network analysis reveals open forums and echo chambers in social media discussions of climate change. Global Environmental Change 32: 126-138.

Whitmarsh, L., and A. Corner (2017). Tools for a new climate conversation: A mixed-methods study of language for public engagement across the political spectrum. Global Environmental Change 42: 122-135.

Xie, J., S. Sreenivasan, G. Korniss, W. Zhang, C. Lim, and B. K. Szymanski (2011). Social consensus through the influence of committed minorities. Physical Review E 84, 011130

Xie, Z. and X. Song (2016). A review of opinion dynamics. AsiaSim 2016, SCS AutumnSim 2016: Theory, Methodology, Tools and Applications for Modeling and Simulation of Complex Systems. Springer, Berlin, pp 349-357.

Zhao, X., Maibach, E., Gandy, J., Witte, J., Cullen, H., Klinger, B.A., Rowan, K.E., Witte, J., and Pyle, A. (2014). Climate change education through tv weathercasts: Results of a field experiment. Bulletin of the American Meteorological Society 95, 117-130.

Zhou, M. (2015). Public environmental skepticism: A cross-national and multilevel analysis. International Sociology 30(1): 61-58. 